\title{
Cerebriform Nevus Sebaceous in a Newborn Infant
}

Authors

\section{Meghmala Sadhukhan $^{1^{*}}$, Baikuntha N. Mishra ${ }^{2}$, Ranjit K. Joshi ${ }^{3}$, Prabin P. Pahi ${ }^{4}$}

${ }^{1}$ Junior Consultant, Department of Paediatrics \& Neonatology, AMRI Hospitals, Bhubaneswar, India

${ }^{2}$ Consultant Paediatric Surgeon, Department of Paediatrics \& Neonatology, AMRI Hospitals, Bhubaneswar, India

${ }^{3}$ Consultant Paediatrician \& Neonatologist, Department of Paediatrics \& Neonatology, AMRI Hospitals, Bhubaneswar, India

${ }^{4}$ Consultant Paediatrician, Department of Paediatrics \& Neonatology, AMRI Hospitals, Bhubaneswar, India

*Corresponding Author

Meghmala Sadhukhan

\begin{abstract}
Nevus sebaceous is a hamartoma of the skin and its adnexa. Cerebriform nevus sebaceous is a morphological variant commonly seen in newborn infants over the scalp as large, pedunculated or verrucous, hairless plaques. Most lesions are isolated but some may be associated with other systemic abnormalities including CNS, cardiovascular, ocular and urological involvement. Most lesions are benign but there is a very small chance of malignant transformation especially when they grow during puberty and hence biopsy should be done in all cases and if possible complete excision.

We describe a newborn infant with cerebriform nevus sebaceous of the scalp with no other systemic abnormalities. The lesion was excised completely and biopsy showed no evidence of malignant potential.

Keywords: Nevus, hamartoma, cerebriform nevus, neonate.
\end{abstract}

\section{Introduction}

Nevus sebaceous (NS) first described by Josef Jadassohn, is a hamartoma of the skin and its adnexa and has been named after him as Nevus Sebaceous of Jadassohn ${ }^{[1]}$. Around 2/3 rd cases of NS are present at birth and the rest appear in the early infantile period ${ }^{[2]}$. Clinically the lesions may be single or multiple and are mostly located on the scalp and face but other sites have also been reported $^{[1],[4],[7]}$. Hair follicles are generally absent over the lesions and sebaceous glands underdeveloped $^{[1]}$. Cerebriform Nevus Sebaceous is a rare morphological variant of NS, named so because of the characteristic morphological appearance with ridges and furrows resembling the surface of the brain ${ }^{[5],[6]}$. Majority of NS are benign but there is a chance of neoplastic transformation in 10 to $30 \%$ cases during puberty when the lesions enlarge $^{[8]}$. There is a small risk of malignant neoplasm in less than $1 \%$ of cases and hence, they should be excised completely if possible ${ }^{[8]-[10]}$. They can be excised in the newborn period for cosmetic reasons with parental consent or followed up till puberty.

Because of its rarity we describe a newborn with giant Cerebriform Nevus Sebaceous who underwent excision biopsy successfully and is doing well on subsequent follow up. 


\section{JMSCR VoI||08||Issue||06||Page 336-340||June}

\section{Case Report}

A 2 day old male baby presented to our hospital with a swelling over the left side of the scalp present since birth. The baby was born in good condition at term by spontaneous vaginal delivery with birth weight of $3.21 \mathrm{Kg}$, in a local hospital and noted to have this swelling since birth for which he was referred for further management. The antenatal period was uneventful with normal antenatal scans.

On examination, a cerebriform plaque -like swelling was noted over the left temporoparietal region of the scalp of size $6 \mathrm{~cm} * 3.5 \mathrm{~cm} * 2.5 \mathrm{~cm}$, raised from the surface, irregular, brownish coloured, soft in consistency and painless, devoid of any hair follicles (Fig 1). Rest of the systemic examination was within normal limits.

MRI brain revealed a lobulated T1 intermediate and T2 hyperintense lesion measuring $24 * 10 \mathrm{~mm}$ in the scalp on the left high parietal region without any flow voids suggestive of slow flow vascular malformation. No intracranial extension was noted. Rest of the brain was normal structurally.

Excisional biopsy of the lesion was planned after consent from parents. Pre operatively all necessary investigations were done. A cerebriform mass measuring about $6 \mathrm{~cm} * 3.5 \mathrm{~cm} * 2.5 \mathrm{~cm}$ on the left temporoparietal region of scalp was excised in toto. Primary suturing was done. No skin graft was needed.

Prophylactic intravenous antibiotics (Ampicillin and Gentamycin) were started preoperatively and continued for a total of 5days and then changed to oral Cefpodoxime for another 5days. Histopathology confirmed the microscopic diagnosis of Nevus sebaceous of Jadassohn. No atypia or malignancy was identified. Baby had uneventful recovery and was discharged home after 10days with advice to continue topical antibiotic over the operative site for another 7days. Follow up review was after one month and the baby was doing well.

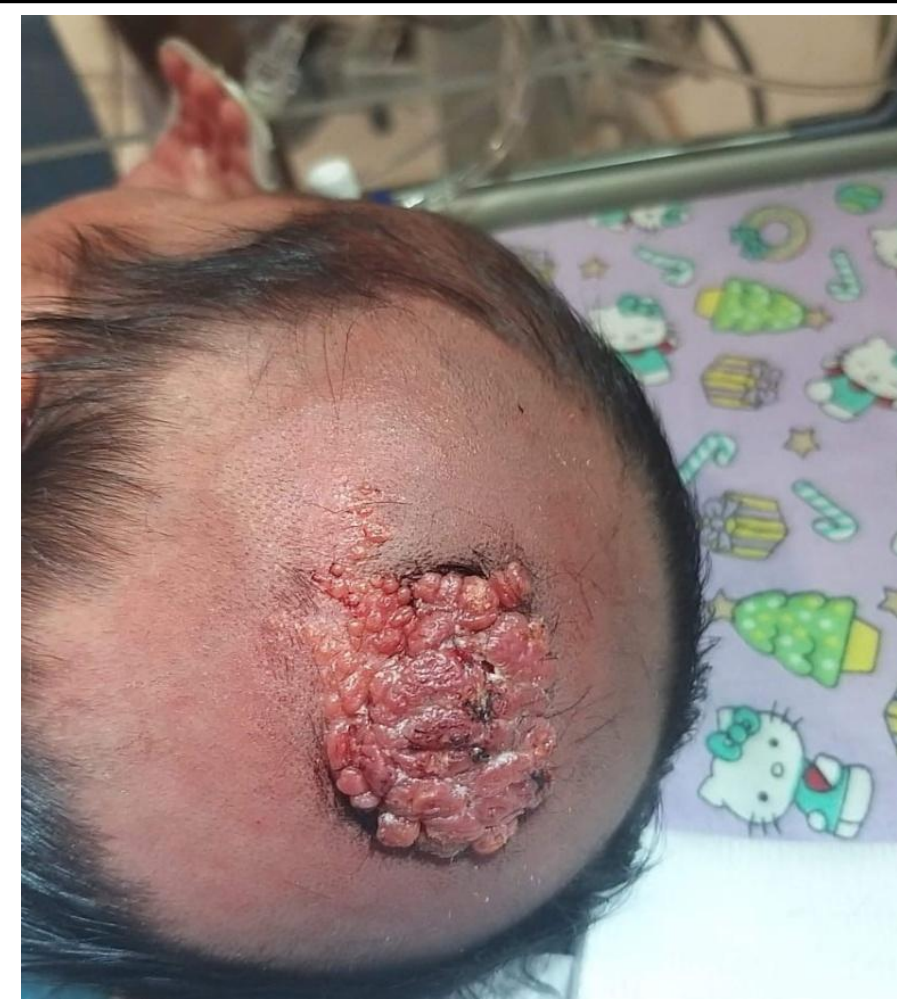

Fig.1 Cerebriform plaque- like swelling over left temporoparietal region of the scalp measuring $6 \mathrm{~cm} * 3.5 \mathrm{~cm} * 2.5 \mathrm{~cm}$, raised from the surface, irregular, brownish colour, painless and devoid of hair follicles.

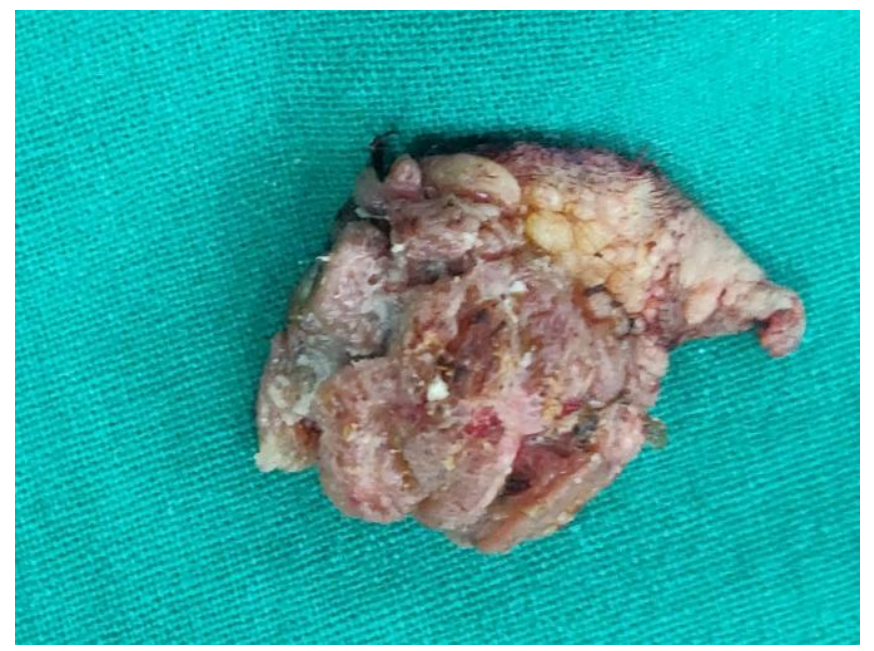

Fig 2 The Cerebriform mass excised in toto.

\section{Discussion}

Nevus Sebaceous (NS) is a hamartoma characterized by epidermal, follicular, sebaceous and apocrine gland abnormalities ${ }^{[1]}$. The condition was first described by Josef Jadassohn, a German dermatologist in 1985 as a circumscribed hamartomatous lesion predominantly formed of sebaceous glands ${ }^{[2]}$. The estimated incidence is $0.3 \%$ 
in newborn infants ${ }^{[1]}$. There is no gender, racial or ethnic predominance ${ }^{[2]}$.

Exact etiology is not known but recent studies suggest a possible link with mothers positive for human papillomavirus ${ }^{[3]}$. The occurrence of the nevus is usually sporadic, although an autosomal dominant mode of inheritance has also been described $^{[3]}$. Deletions in the PTCH tumour suppressor gene on chromosome 9p22.3 may account for the neoplastic potential of the hamartoma ${ }^{[8]}$.

Approximately two thirds of cases are present at birth and the rest develop in early childhood. At birth, nevus sebaceous typically presents as a solitary, well-circumscribed, smooth to velvety, yellow to orange, round, oval, or linear, flat or minimally raised, plaque ${ }^{[2],[3]}$. The scalp and face are sites of predilection, although the trunk and extremities may also be affected ${ }^{[1]-[3]}$. Lesions on the scalp are typically hairless and may be pedunculated ${ }^{[1-3]}$. The lesions are often distributed along Blaschko's lines and are arrayed in a linear configuration. Characteristically, the lesion grows proportionally with the size of the patient and then grows more rapidly, becomes more thickened and protuberant, and at times acquires a verrucous or even a nodular appearance ${ }^{[1],[2]}$. Nevus sebaceous is usually solitary, although multiple and widespread lesions have been reported ${ }^{[4]}$.

A new phenotype, known as cerebriform nevus sebaceous was first described by Ramesh et al. in 1998, when they described a 20-year-old man with a large cerebri-form plaque over the right parietooccipital region, which was confirmed by histopathological examination ${ }^{[5]}$. After that, there were reported cases with similar presentation in different areas $^{[6]}$. Cerebriform nevus sebaceous is characterized by large, pedunculated or verrucous, pink, hairless, nodules or tumors in newborn infants [5],[6].

Nevus sebaceous is usually an isolated finding but it may sometimes be associated with syndromes like SCALP syndrome (sebaceous nevus, central nervous system malformation, aplasia cutis congenita, limbal dermoid, and pigmented nevus) and linear

sebaceous

nevus

syndrome

(Schimmelpenning syndrome) characterized by extensive nevus sebaceous distributed along Blaschko's lines, central nervous system abnormalities, epilepsy, mental retardation, ocular abnormalities, musculoskeletal defects, cardiovascular abnormalities, and urologic abnormalities $^{[4],[7]}$. Large or extensive lesions may be associated with developmental deficits ${ }^{[4]}$. In our case, the systemic examination of the newborn was within normal limits and MRI brain did not show any other associated CNS anomalies.

Nevus sebaceous should be differentiated both clinically and histopathologically from epidermal nevus, aplasia cutis congenita, solitary mastocytoma, and juvenile xanthogranuoloma.

The diagnosis is usually clinical based on the characteristic features. Neuroimaging like CT or MRI brain may help to diagnose associated CNS anomalies. The final diagnosis is made by histopathology. Histopathology includes early infantile stage characterized by papillomatous epithelial hyperplasia ${ }^{[8]}$. The epidermis is only slightly acanthotic ${ }^{[8]}$. The hair follicles are underdeveloped and the sebaceous glands are not prominent ${ }^{[1],[8]}$. Apocrine glands are rarely seen ${ }^{[1]}$. During puberty, sebaceous glands become numerous and hyperplastic, apocrine glands become hyperplastic and cystic, and the epidermis becomes verrucous $^{[1],[8]}$. The hair follicles remain small and primordial and may disappear altogether ${ }^{[1],[8]}$. During adulthood, epidermal hyperplasia, large sebaceous glands, and ectopic apocrine glands are characteristic histological findings ${ }^{[8]}$. Normal hair follicles are characteristically absent ${ }^{[9]}$. This stage is also distinguished by the potential development of a variety of adnexal tumours. There is an increased expression of androgen receptors in nevus sebaceous ${ }^{[9]}$. The growth of the lesion in adolescence is due to increased production of androgen at the time of puberty and its influence on the pilosebaceous and apocrine elements ${ }^{[9]}$.

Nevus sebaceous may be complicated by the development of benign and malignant nevoid tumors in the original nevus ${ }^{[8]-[10]}$. The incidence of 
these tumors increases with age, particularly after puberty ${ }^{[10]}$. Neoplasms occur mostly in the fourth decade of life in approximately 10 to $30 \%$ of lesions ${ }^{[8]}$. Majority of these tumors are benign; less than $1 \%$ of nevus sebaceous is complicated by malignant tumors $^{[8]}$. Malignancy is suggested by the acute appearance of a large, discrete, ulcerating nodule within the lesion ${ }^{[9]}$. The most common benign tumors are syringocystadenoma papilliferum followed by trichoblastoma ${ }^{[10]}$. The most common malignant tumor is basal cell carcinoma ${ }^{[10]}$.

For cosmetic purpose complete excision can be considered at any age. However, because of the potential of malignant transformation, some authors recommend prophylactic full-thickness, complete excision of the lesion with minimum 2 to $3 \mathrm{~mm}$ margins ${ }^{[1],[2]}$. In large lesions tissue expanders may be required for primary closure and in some cases a skin graft may be required to to cover the defect. In our case full excision of the lesion was done successfully and the biopsy results showed no evidence of malignancy.

Ablative laser and photodynamic therapy may be considered for the occasional cases with inoperable lesions ${ }^{[10]}$. Surgery is deferred in most cases till adolescence because of its benign nature and the risk of anaesthesia in the neonatal period ${ }^{[10]}$. However, surgery is feasible in the neonatal period in a good tertiary neonatal surgical centre if the lesion is operable without the requirement of skin grafting. In our case, complete full thickness excision was done successfully and wound closed primarily without any complications.

\section{Conclusion}

Cerebriform Nevus sebaceous is a rare condition in a neonate. It may be associated with other congenital anomalies; hence all cases should be examined clinically well and necessary investigations undertaken. Although a hamartomatous lesion, it has a very small malignant potential, hence complete excision should be done in cases which are operable and also for cosmetic reasons.

\section{Acknowledgement}

Concept and co ordination for the manuscript: Baikuntha N. Mishra

Data acquisition and manuscript preparation: Meghmala Sadhukhan, Baikuntha N. Mishra

\section{Source of funding: None}

\section{Reference}

1. Terenzi V, Indrizzi E, Buonaccorsi S, Leonardi A, Pellacchia V, Fini G. Nevus sebaceus of Jadassohn. J Craniofac Surg. 2006; 17: 1234-1239.

2. Saedi T, Cetas J, Chang R, Krol A, Selden NR. Newborn with sebaceous nevus of jadassohn presenting as exophytic scalp lesion. Pediatr Neurosurg. 2008; 44: 144147.

3. West C, Narahari S, Kwatra S, Feldman S. Autosomal dominant transmission of nevus sebaceous of Jadassohn. Dermatol Online J. 2012; $18: 17$.

4. Chi SG, Kim JY, Kim HY, Lee SJ, Kim do W, Lee WJ. Multiple nevus sebaceous occurring on the scalp and on the contralateral side of the face. Ann Dermatol. 2011; 23: 389-391.

5. Ramesh A, Murugusundaram S, Vittel K, Kumar S, Janaki VR, Boopalraj JM: Cerebriform sebaceous nevus. Int $\mathbf{J}$ Dermatol 1997;37:220.

6. Valerio E, Mardegan V, Zanella C, Gaio P, Cutrone M: Cerebriform nevus sebaceous in neonate. J Pediatr 2014;164:665-666.

7. Menascu S, Donner EJ: Linear nevus sebaceous syndrome: case reports and review of the literature. Pediatr Neurol 2008;38:207-210.

8. Altaykan A, Ersoy-Evans S, Erkin G, Ozkaya O. Basal cell carcinoma arising in nevus sebaceous during childhood. Pediatr Dermatol. 2008; 25: 616-619.

9. Simi CM, Rajalakshmi T, Correa M. Clinicopathologic analysis of 21 cases of nevus sebaceus: a retrospective study. Indian 
J Dermatol Venereol Leprol. 2008; 74: 625627.

10. Idriss MH, Elston DM. Secondary neoplasms associated with nevus sebaceus of Jadassohn: a study of 707 cases. J Am Acad Dermatol. 2014; 70: 332-337. 